\title{
Preparation of Ultra-thin Carbon Supporting Film for HRTEM of Nano- particles
}

\author{
Y-M Kim, J-S Kang, J-M Jeung, Y-C Ko and Y-J Kim \\ Division of Nano-Material \& Environmental Sciences, Korea Basic Science Institute, Daejeon, \\ 305-333, Korea
}

A simple preparation method of ultra-thin C-supporting film especially useful for HRTEM of nano-particles is introduced. In order to obtain high resolution electron micrographs including fine structures of individual particles as well as large-scale structures of self-assembling particles, interference of the supporting film on the image must be minimized and the thin film area should be maximized. Although there are some commercial grids and the methods to prepare for the ultra-thin C-supporting film have been reported [1] [2], it is difficult for these grids to obtain both types of electron micrographs for the nano-particles having a strong magnetic property. Oxidized iron nano-particles were used as a testing sample in this experiment. Overall aspects of the ultrathin C-supporting film have been acquired by using a HVEM (JEM-ARM1300S, JEOL). Some of the film qualities have been discussed with the results of EELS and image analysis techniques such as thickness maps and histograms by using a post column imaging filter (HV-GIF, Gatan). Comparison of the film qualities between the homemade and the commercial ones have also been carried out.

A conventional method to fabricate the holey carbon film was modified to form larger ultra-thin C-supporting area over the hole by the detention time $(10 \sim 20 \mathrm{~s})$ in the procedure, where it is to form droplets of water on the hydrophobic glass slide over the ice pack. The main idea was to utilize the carbon film lying over the holes which should be the thinnest supporting layer available for high resolution electron microscopy. Highly consistent results were achieved by that the micronet are prepared by immersing slides into a solution of $0.25 \mathrm{vol} \%$ formvar in chloroform and then slightly coated with carbon. No strict processing conditions were required since this process was very reproducible for ambience conditions of $20 \sim 28^{\circ} \mathrm{C}$ room temperature and $30 \sim 70 \%$ humidity.

As shown in Fig. 1, it has been demonstrated that ultra-thin C-supporting film over the hole is sufficient to image the large-scale assembly of the nano-particles. In addition, HRTEM images show more clearly fine structures of individual particles, as compared to the case of the commercial grid. The histogram analysis in Fig. 2(a), higher electron counts and narrower width of histogram in the homemade film with same acquisition condition, indicates that the image has higher contrast and brightness. The relative thickness of C-supporting film is 0.6 time less than that of the commercial one calculated from the EELS. It can be seen in Fig. 2(b) that the thicknesses of both $\mathrm{C}$-supporting films vary within about 3\%. This thickness variation of $\mathrm{C}$ supporting films was obtained from the logarithmic ratio of images derived from $-\ln \left(1+\mathrm{I}_{\mathrm{o}} / \mathrm{I}_{\mathrm{p}}\right)$, where $I_{o}$ is the zero-loss image and $I_{p}$ is the first plasmon-loss image of the same area. It is assumed that the higher thickness variation in the homemade film would not obviously produce uneven contrast in HRTEM image due to its reduced thickness.

[1] S. Iijima, Micron 8(1/2) (1977) 41.

[2] R. Reichelt et al., Micron 8(1/2) (1977) 29. 

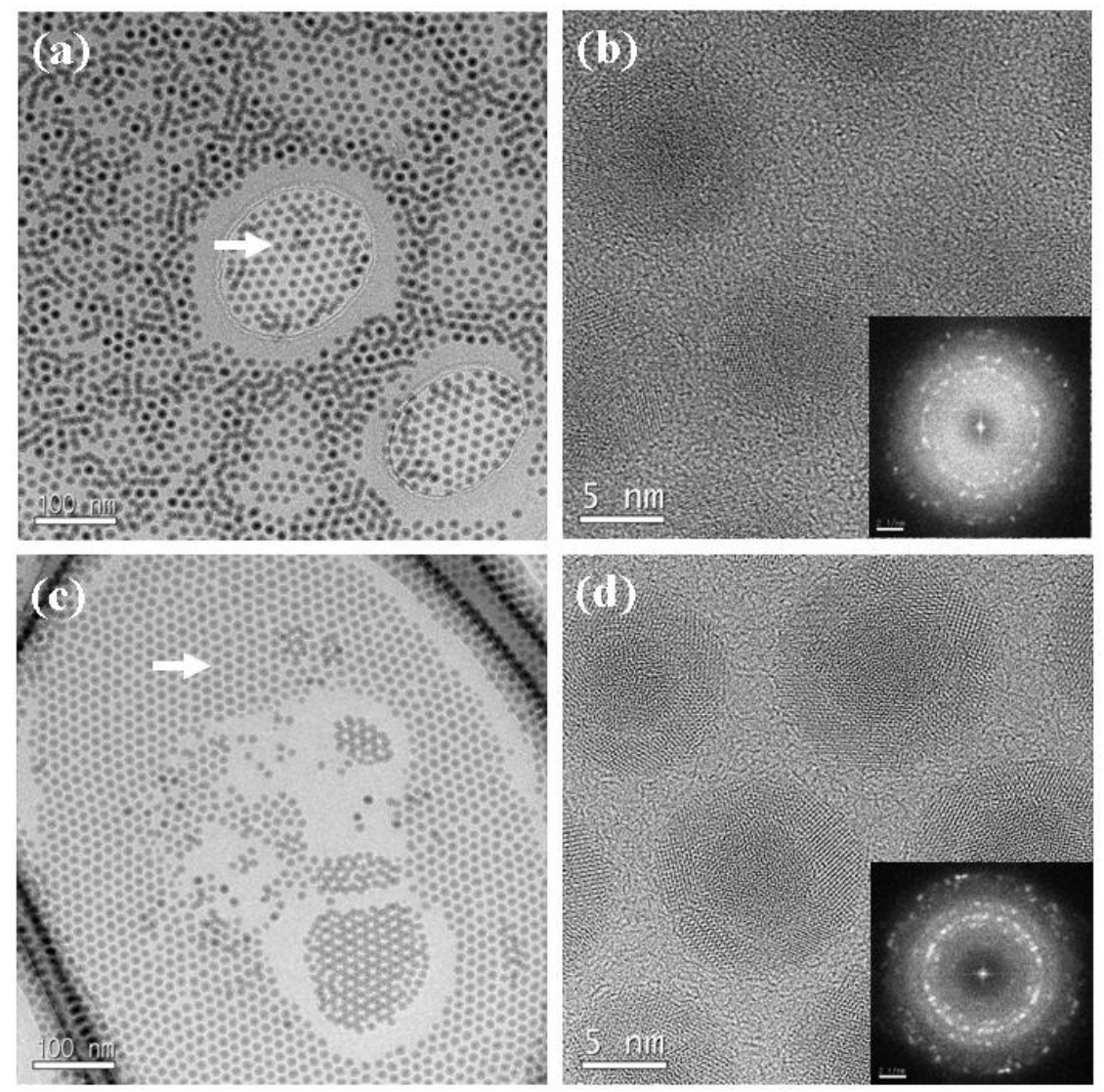

Fig. 1. TEM images of oxidized iron nano-particles lying on the commercial ultra-thin holey Cgrid (a) and the homemade grid (c). HRTEM images, (b) and (d), of the areas in (a) and (c) indicated by white arrows in the C-supporting film lying over the hole.
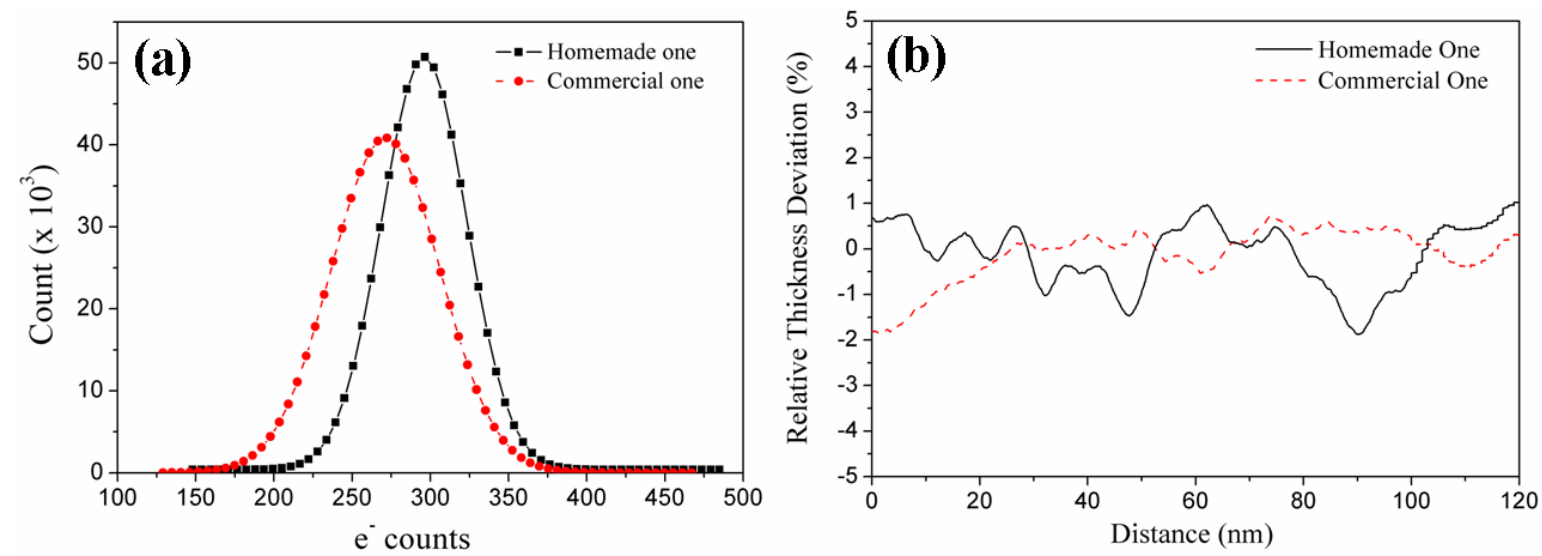

Fig. 2. Comparison of the commercial and homemade grids: (a) the histogram results from electron counts in the images; (b) relative film thickness variations of the ultra-thin carbon films corresponding to intensity profiles across the horizontal distance in the thickness map images. 